\title{
Plasmonic response in nanoporous metal: dependence on network topology
}

\author{
Marc A. Galía ${ }^{\text {, Matthew C. Tai }}{ }^{\mathrm{a}}$, Matthew D. Arnold ${ }^{\mathrm{a}}$, Michael B. Cortie ${ }^{\mathrm{a}}$, Angus R. Gentle ${ }^{\mathrm{a}}$, \\ and Geoffrey B. Smith ${ }^{a}$ \\ ${ }^{a}$ Institute for Nanoscale Technology, University of Technology Sydney, PO Box 123 Broadway \\ NSW 2007, Sydney, Australia.
}

\begin{abstract}
The optical and electrical responses of open, nanoscale, metal networks are of interest in a variety of technologies including transparent conducting electrodes, charge storage, and surfaces with controlled spectral selectivity. The properties of such nanoporous structures depend on the shape and extent of individual voids and the associated hyper-dimensional connectivity and density of the metal filaments. Unfortunately, a quantitative understanding of this dependence is at present only poorly developed. Here we address this problem using numerical simulations applied to a systematically designed series of prototypical sponges. The sponges are produced by a Monte Carlo simulation of the dealloying of $\mathrm{Ag}-\mathrm{Al}$ alloys containing from $60 \%$ to $85 \% \mathrm{Al}$. The result is a series of $\mathrm{Ag}$ sponges of realistic morphology. The optical properties of the sponges are then calculated by the discrete dipole approximation and the results used to construct an 'effective medium' model for each sponge. We show how the resulting effective medium can be correlated with the associated morphological characteristics of each target and how the optical properties are primarily controlled by the density of the sponge and its state of percolation.
\end{abstract}

Keywords: optical properties, nanoporous sponge, metamaterials, silver, effective media

\section{INTRODUCTION}

The interaction of light with materials is influenced by their dielectric function (an intrinsic property) and their geometry (including shape, morphology or structure, all extrinsic properties). At the nanoscale (i.e. at a size range well below the wavelength of light) dielectric function and geometry become intertwined and the resulting properties are often expressed as an 'effective medium' which takes geometry into account. ${ }^{1}$ The transition from a network with weak metal connectivity and more localized optical response to one with macroscopic percolation and delocalized response involves a sequence of morphologies. Signature topological features emerge near the percolation threshold but there is usually a broad admix of local void topologies within any sponge making analysis complex. The fine detail of geometry is thus lost in the formulation of the effective medium. Explicit consideration of the geometry of the material would give an extra order of precision in these models but the problem is intractable from an analytical viewpoint.

In particular, it would be of particular benefit to model nanoporous metallic sponges using an 'effective medium' approach. Nanoporous metals are of interest because they can be used in a wide range of applications, including as capacitive sensors, actuators, chemical catalysts, electrodes for supercapacitors of Li-ion cells, or as surface coatings with controlled optical properties. ${ }^{2-6}$ It is this latter application that is relevant here: in the present work we investigate the correlation between the morphology and the optical properties of sponges. To do so, we will study a silver $(\mathrm{Ag})$ sponge-like metamaterial of the form typically produced by de-alloying $\mathrm{Ag}-\mathrm{Al}$ precursor alloys. Here we generate model versions of these sponges by a numerical process that simulates de-alloying and atomic rearrangement. It has been shown elsewhere that the model successfully reproduces the classic bicontinuous vermicular sponges that are frequently observed in noble metals. ${ }^{7}$ The sponges are then used as targets for the calculation of the optical properties using the discrete dipole approximation (DDA) and the resulting optical properties used, in turn, to construct an 'effective medium approximation' (EMA) for the material. We then explore how these EMAs are related to the morphology of each type of sponge. Finally, we will invert the EMA to deduce the optical properties of Au sponge.

Further author information: (Send correspondence to G.B.S.)

G.B.S.: E-mail: g.smith@uts.edu.au

SPIE Micro+Nano Materials, Devices, and Systems, edited by Benjamin J. Eggleton, Stefano Palomba Proc. of SPIE Vol. 9668, 96683S - @ 2015 SPIE · CCC code: 0277-786X/15/\$18 · doi: 10.1117/12.2202278

Proc. of SPIE Vol. $966896683 S-1$ 


\section{METHODOLOGY}

\subsection{Simulation of sponges}

In this work we generated a series of nanoporous sponges using a code that simulates the dealloying of an alloy between a noble metal and an active metal (see elsewhere for a description of the code ${ }^{7,8}$ ). In our case the noble element was silver $(\mathrm{Ag})$ and the active, sacrificial, element was aluminum (Al). Elapsed time in the simulations is measured in 'sweeps', with one sweep defined as the completion of the same number of Monte Carlo iterations as there are atoms in the simulation. Therefore, on average, each atom has been visited once after one sweep has been completed. The dealloying part of the simulation finishes when all $\mathrm{Al}$ is gone from the sponge, and at this point we reset the sweep counter to 0 and anneal the target for 15,000 sweeps. During this annealing phase thermally-activated atomic movements driven by a Metropolis Monte Carlo algorithm operating with a Lennard-Jones interatomic potential reshape the sponge. The process leads to coarsening of the ligaments and is effectively being driven by the intrinsic surface tension of the Lennard-Jones solid. Finally, we machine a spherical shape from each cuboid of sponge, for use in the optical property calculations. As we will show, use of a spherical target brings the distinct advantage that the analytical Mie solutions can be applied within the analysis. The sponges are labeled according to their initial $\mathrm{Al}$ content, and the number of sweeps to which they had been coarsened.

The spherical cutout is performed on structures recorded at selected sweep numbers chosen so that the intervals are approximately evenly spaced on a logarithmic time axis. Consequently, the amount of Ag inside the ball of sponge after additional sweeps can vary slightly, since although we cut it in the same region every time the silver atoms can move a little between sweeps. Therefore, there is a small flux of Ag atoms passing in or out of the spherical template used to machine the ball of sponge. The second implication of our method is related to how we cut the cube; in order to be more realistic, the method that we are using to simulate the dealloying and coarsening process works in real space $\left(\mathbb{R}^{3}\right)$, i.e. it is a so-called 'off-lattice' algorithm and the position of each atom is expressed using floating point number. In contrast, the computer code we used to calculate the optical properties (see section 3.1), needs a target where the dipoles have integer coordinate positions, i.e. are in $\mathbb{Z}^{3}$. There are small computational discrepancies when a $\mathbb{R}^{3}$ sponge is converted to an array of dipoles in $\mathbb{Z}^{3}$, and further small errors when we fix a radius and proceed with cutting the sphere shape out of the block of sponge (in the latter case, dipoles near the sphere surface have to fall completely inside or outside the cutout and there will be some stochastic variation). Consequently, the actual radii of the resulting spheres vary slightly from the nominal value of $30 \mathrm{~nm}$. These small errors will of course propagate through to the effective medium approximation (EMA) that we will be deriving.

\subsection{Effective media approach}

There are several models that aim to provide a quantitative understanding of the optical properties of mesoporous materials, ${ }^{9-11}$ but in our opinion none of them is fully satisfactory yet. Here we will exploit a new approach to develop an Effective Medium Approximation (EMA) of the sponges. Essentially, our aim is to define a homogeneous virtual material that has the same extinction cross section and same phase delay cross section as the complex sponges under study. To achieve this, we will use the extinction and phase efficiency calculated by DDSCAT to derive the complex dielectric function (or, equivalently, the refractive index) of the effective medium.

The desired extinction and phase cross sections are defined as followed: ${ }^{12,13}$

$$
\begin{aligned}
C_{e x t} & =\frac{4 \pi k}{\left|E_{i n c}\right|^{2}} \sum_{i=1}^{n} \operatorname{Im}\left(E_{i n c, i}^{*} \cdot P_{i}\right), \\
C_{p h a} & =\frac{2 \pi k}{\left|E_{i n c}\right|^{2}} \sum_{i=1}^{n} \operatorname{Re}\left(E_{i n c, i}^{*} \cdot P_{i}\right) .
\end{aligned}
$$

$C_{e x t}$ gives the energy loss caused by the combination of scattering and absorption. On the other hand, $Q_{p h a}=\frac{d C_{p h a}}{d \Omega}$ is a factor that is related to the phase lag due to the effective medium, and consequently is directly related to the real part of the polarizability, $\alpha$. In principle this quantity can also be measured directly 
by interferometry or indirectly by ellipsometry. Note that in the literature there is a difference of a factor 2 in the definition of this parameter, however, as we are using the simulated data from DDSCAT, to be internally consistent, we will use the definition given by Draine ${ }^{12}$ (eq. 2).

First of all, we will work with the expressions of $C_{e x t}$ and $C_{p h a}$ (eq. 1 and 2) in order to define the effective polarizability. Therefore, as we are assuming that our effective medium is homogeneous, previous relations, equations 1 and 2 simplify since the polarizability tensor becomes diagonal (assuming either isotropy or alignment of optical and material axes), i.e. $\alpha_{i, j}=0$ if $i \neq j$, and $\alpha_{i, i}=\alpha$. Then, using the relationship $P=\alpha E_{i n c}$, the previous equations become:

$$
\begin{aligned}
C_{e x t} & =4 \pi k \operatorname{Im}(\alpha), \\
C_{p h a} & =2 \pi k \operatorname{Re}(\alpha),
\end{aligned}
$$

where $k \equiv 2 \pi / \lambda$ is the wave number. Let be $C^{S}$ and $C^{e f f}$ the sponge and effective medium cross section factors, then imposing our EMA, i.e. $C^{S} \equiv C^{e f f}$, the efficiencies are related as follows:

$$
Q^{e f f} \equiv\left(\frac{a_{e f f}}{R}\right)^{2} Q^{S}
$$

where $Q^{S}$ and $Q^{e f f}$ are the sponge and the effective medium efficiencies, respectively. Then, we can define the effective polarizability in terms of the efficiencies of our sponge.

$$
\begin{aligned}
\alpha^{e f f} & =\frac{Q_{p h a}^{e f f}}{2 x}+\frac{Q_{e x t}^{e f f}}{4 x} \mathbf{i} \\
& \equiv\left(\frac{a_{e f f}}{R}\right)^{2}\left(\frac{Q_{p h a}^{S}}{2 x}+\frac{Q_{e x t}^{S}}{4 x} \mathbf{i}\right)
\end{aligned}
$$

where $x=2 \pi R \epsilon_{0}^{1 / 2} / \lambda$ is the scale parameter.

Finally, we can use Claussius-Mossotti approximation to relate the polarizability to the permittivity. Thus, we obtain the following expression:

$$
\epsilon=\epsilon_{0} \frac{2 \alpha+1}{1-\alpha} .
$$

\section{RESULTS}

\subsection{Optical properties}

Six different model precursors with starting $\mathrm{Al}$ concentrations of $60 \%, 65 \%, 70 \%, 75 \%, 80 \%$ and $85 \%$ (atomic percentages) were dealloyed, and each then subjected to 15000 sweeps of coarsening under the action of the model. Intermediate structures were recorded at 0, 100, 200, 500, 2000, 5000 and 15000 sweeps. Thus, we study $6 \times 7$ different Ag sponges. Representative examples are shown in Figure 1. It can be seen that increasing the $\mathrm{Al}$ content of the precursor results in a loss of percolation and decrease in density of the resulting $\mathrm{Ag}$ sponge. In addition, prolonged application of the Monte Carlo simulation results in the growth and thickening of the ligaments of the sponge.

The calculated optical properties are shown in Figure 2 in which it can be seen that the sponges practically do not scatter, i.e. the extinction is mainly dominated by the absorption $\left(Q_{e x t} \sim Q_{a b s}\right)$ - thus the sponges meet a necessary condition for application of any EMA. Also, as the concentration of $\mathrm{Al}$ in the precursor alloy increases the resulting sponges are becoming less absorbent, which is expected since the sponges are becoming less dense. 


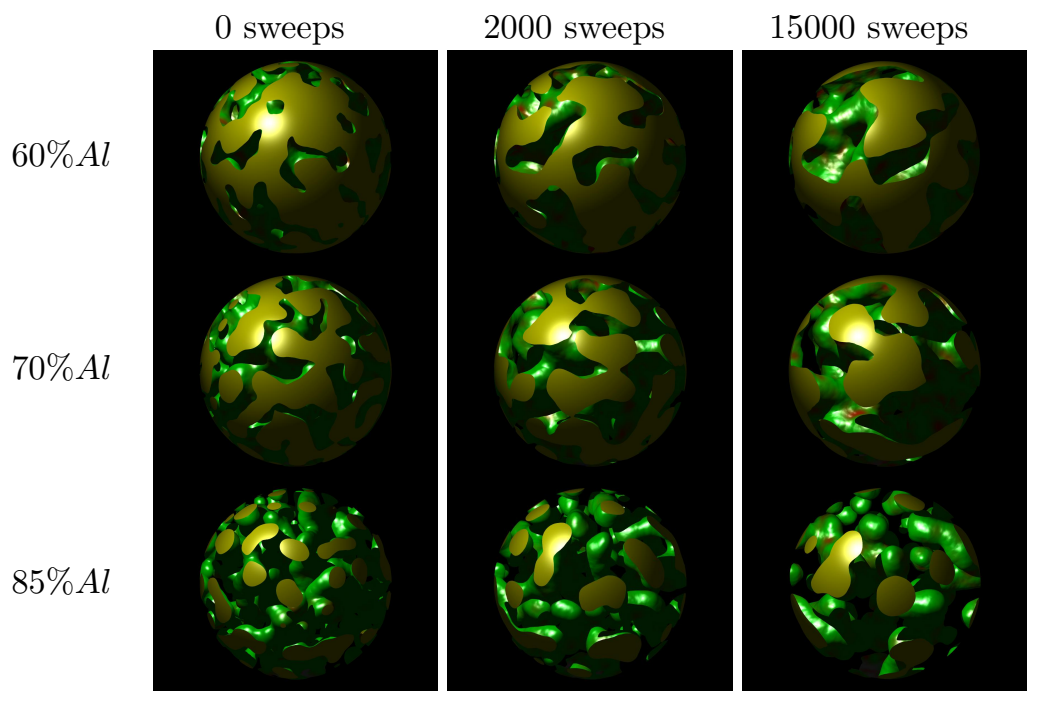

Figure 1. Sponge ball visualization

The distinct extinction peak due to a dipolar plasmon resonance in the sphere is lost as the density of the sponge decreases and this is more clearly shown in the color map of Figure 3.

As we explained in the previous section (sec. 2.2), $Q_{p h a}$ is related with the phase lag, where positive phase efficiency means that the phase velocity $\left(v_{p}\right)$ is smaller than c, and negative $Q_{p h a}$ means $v_{p}>c$, it is worth to note that $v_{p}>c$ only mean the the phase is traveling faster than the light, but not the wavefront.

Phase velocity is defined as $v_{p} \equiv c / n$, i.e. $v_{p}>c$ if the refractive index is smaller than 1 . As our sponges are dispersive media $(n(\lambda))$, the phase velocity will vary with the incident wavelength and thus, the $Q_{p h a}$ will do too. It can be seen in Figure 2, that $Q_{p h a}$ achieves negative values at smaller wavelengths $(<400 \mathrm{~nm})$ and, as expected, that happens in the same wavelength range where $n<1$ (see Figure 6).

\subsection{Effective media}

Effective medium models can only be applied when the size of the original medium is much smaller than the incident wavelength. Thus, the applicability of EMA usually is determined by the wavelength and the scale parameter through the inequality $2 \pi x<<\lambda$. Our simulated samples have a radius $\sim 30 \mathrm{~nm}$ and we are analyzing them for incident wavelength in the visible range $300-700 \mathrm{~nm}$, so we are well inside the boundary conditions. Therefore, it is reasonable to apply an EMA.

It is important to note that the generation of the spherical sponges implies another problem, as we did not dealloyed the porous sphere, instead we cut it, our samples have a very rough surface (especially the sponges with low concentration of silver) and in these samples the outermost region of the ball sponge are almost unpercolated. This means that the grade of connectivity of the outermost part ('shell') of the sphere is not the same than the core, i.e. the 'shell' of the sphere has not the representative topology of the porous sphere. This may arise another issue, that we will not study further on in the present work, if the skin depth is smaller than the thickness of the sphere shell, we will only be analyzing the optical response of the shell, which is not the topological representative part of the sample.

Using the approximation explained before (section 2.2), and the data that we calculated for our sponges we found a spherical effective medium, Figures 4 to 6 . It is important to emphazise two points; our effective media model is useful for directly analyzing the equivalent refractive index or dielectric permittivity of each sponge, and so, provide insights into fundamental trends of the sponge spectra. Nevertheless, specific information about localized electric fields or plasmon resonances of the porous samples cannot be found from the EMA. Indeed, as demonstrated in literature, ${ }^{3,14-16}$ the localized plasmon resonances are very sensitive to structural irregularities and may be present at a size scale of the order of several nanometers (i.e. much smaller than the sphere of 

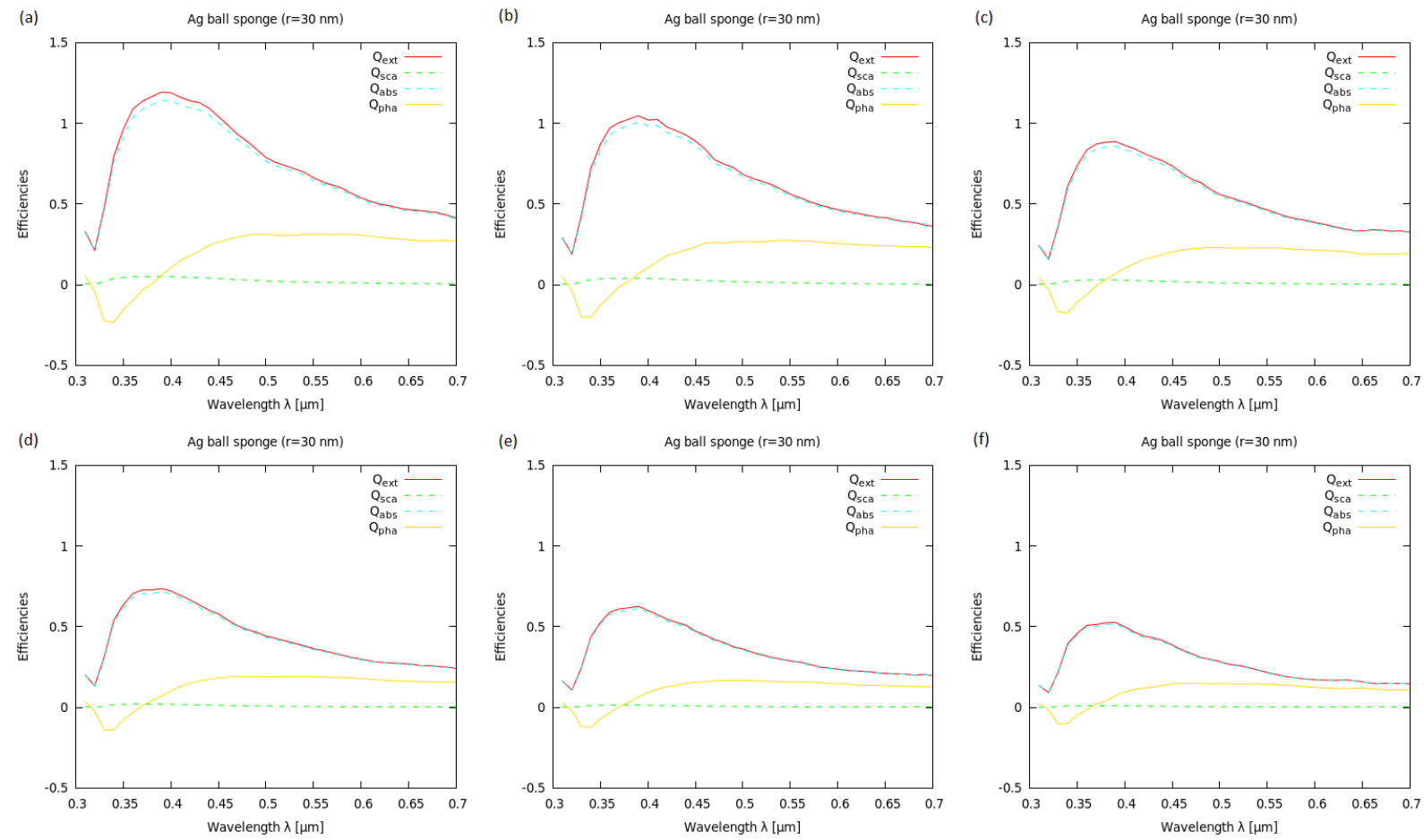

Figure 2. Normalized efficiencies (cross section equals to $\pi 30^{2} \mathrm{~nm}^{2}$ ) at 0 sweeps of a sponge with an initial Al fill factor of (a) $60 \%$, (b) $65 \%$, (c) $70 \%$, (d) $75 \%$, (e) $80 \%$ and (f) $85 \%$.

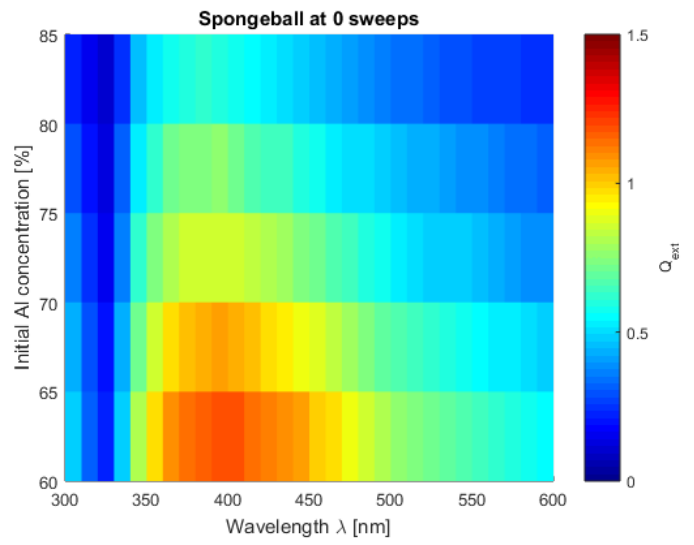

Figure 3. Extinction efficiency of a spherical sponge in terms of the initial concentration of aluminum in the precursor alloy. To a first order approximation the latter quantity can be taken as the void fraction.

sponge). One implication of this is that plasmon resonances of the original cubic sponge will not be the same as the resonances of the spherical sponge. The original sponge has been generated with periodic boundaries in the horizontal plane, i.e. the structure is topologically equivalent to an infinite plane, and a structure such this generates a surface polariton current. ${ }^{17,18}$ Nonetheless, after the cutting we obtained a small sphere (compared to the incident wavelength) and this structure is more likely to create a localized surface plasmon (LSP). Thus, with this method the localized plasmon resonance of the sphere surface dominates the optical response, so we can not infer in the plasmon resonance of the porous material.

It can be seen that, as expected, the extinction coefficient decreases as the metal fill factor decreases as well. This is because the sponge becomes less absorbent and scattering. Nonetheless, the behavior of its refractive 

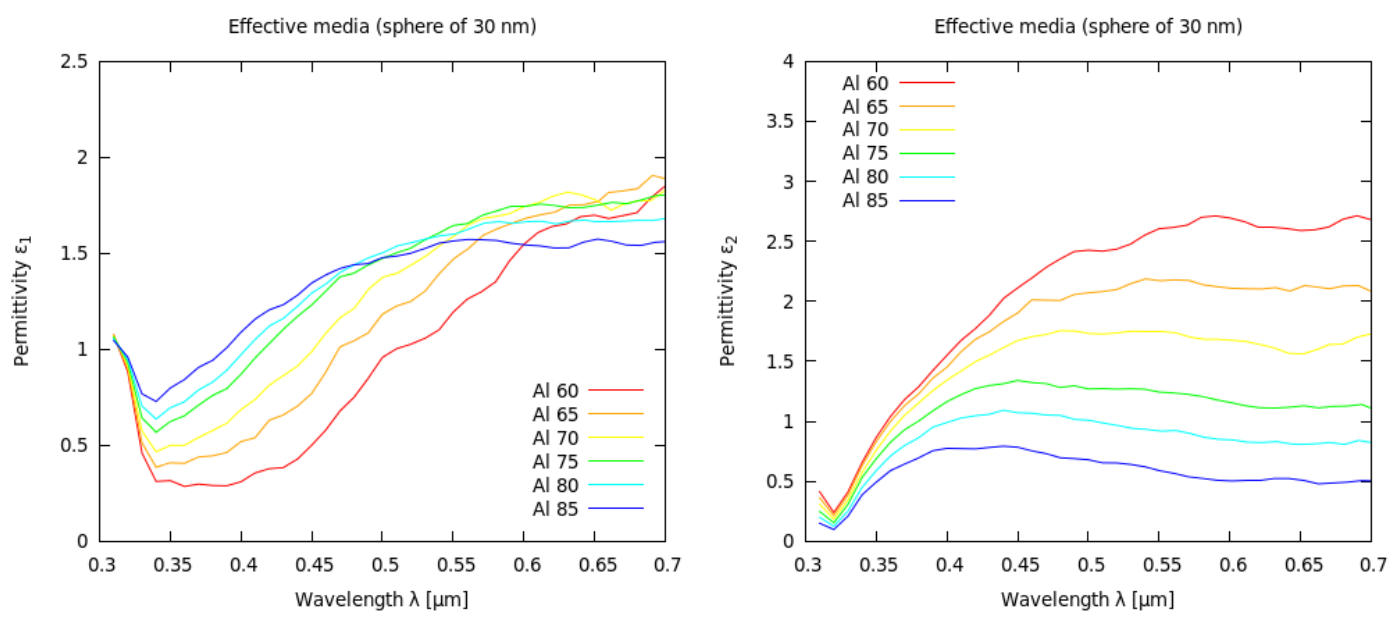

Figure 4. Effective media that has been extracted for different sponges (taken at 0 sweeps of coarsening) in terms of the real part (left) and imaginary part (right) of the dielectric function.
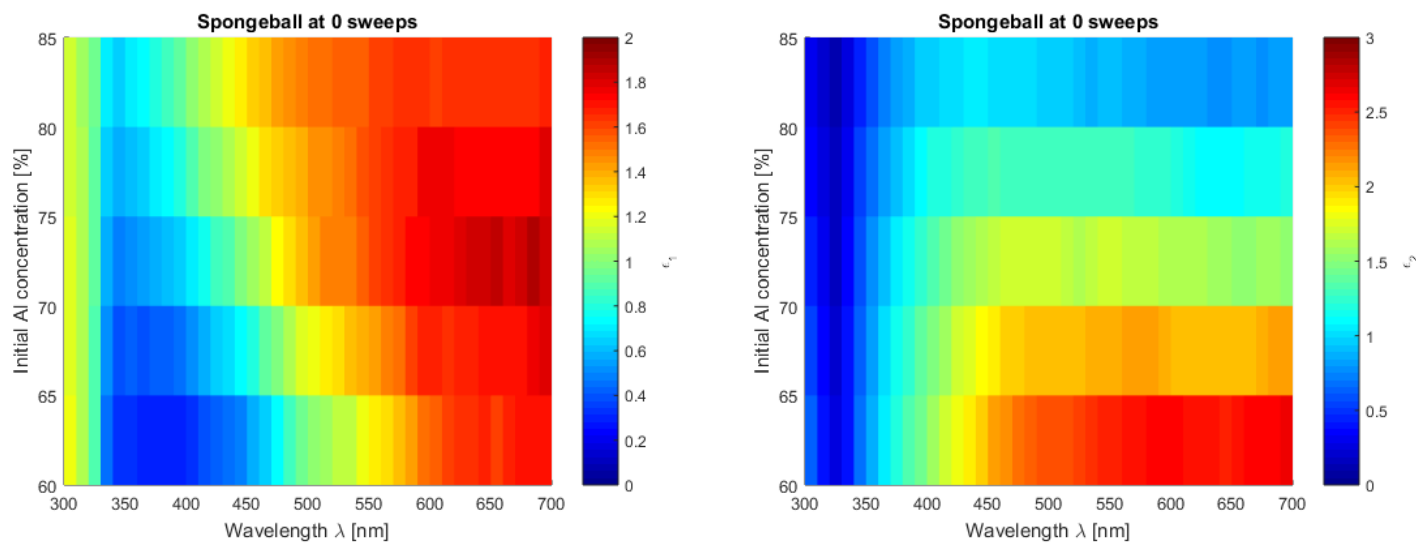

Figure 5. Real and imaginarypart of the permittivity at 0 sweeps.

index $(n)$ is more interesting because at the shorter wavelengths $(\sim 300-350 \mathrm{~nm})$ the response of each sponge is closer to bulk silver, Figure 7. However, for greater wavelengths $(>350 \mathrm{~nm})$ the topology of the sponge starts to play a dominating role. Due to the low amount of Ag, instead of having a solid sphere with voids inside (concave metal structure), we have a spherical network of silver filaments (convex metal structure). In this situation, the phase that has a higher grade of connectivity is the void-phase, i.e. the incident wave will mainly travel through vacuum, what implies that our sample would have a higher transmittance and a low energy loss $(k \sim 0)$, Figure 7. Figure 6 shows another interesting aspect of the sponges, the real part of the refractive index and the extinction coefficient acquire an almost constant value at longer wavelengths, i.e. at these wavelengths the trend is similar to the refractive index of vacuum.

As been seen elsewhere ${ }^{19}$ the plasmonic response can be studied analyzing the complex dielectric function. In our samples, overall, the optical responses of the sponges are not strongly plasmonic due to the positive values of the effective $\epsilon_{1}$. This can also be seen in the fact that, in general, the effective refractive index $\mathrm{n}$ is larger than the effective extinction coefficient, k, i.e. $k / n<1$, so they do no behave as metallic sponges. Indeed, can be seen in Figure 4 that $\epsilon_{2} / \epsilon_{1} \sim 1$, so it is more accurate to say that our sponges have a lossy material behavior. 

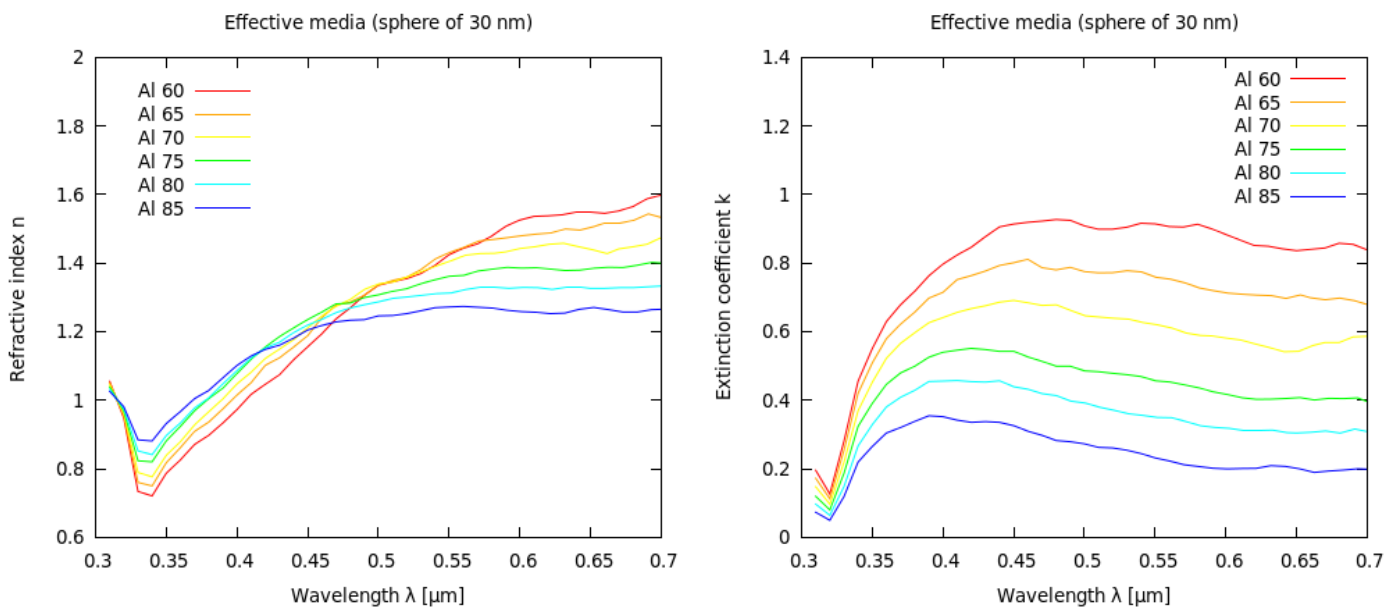

Figure 6. Generated effective media for different concentrations at 0 sweeps in terms of real part (left) and imaginary part (right) of the refractive index.
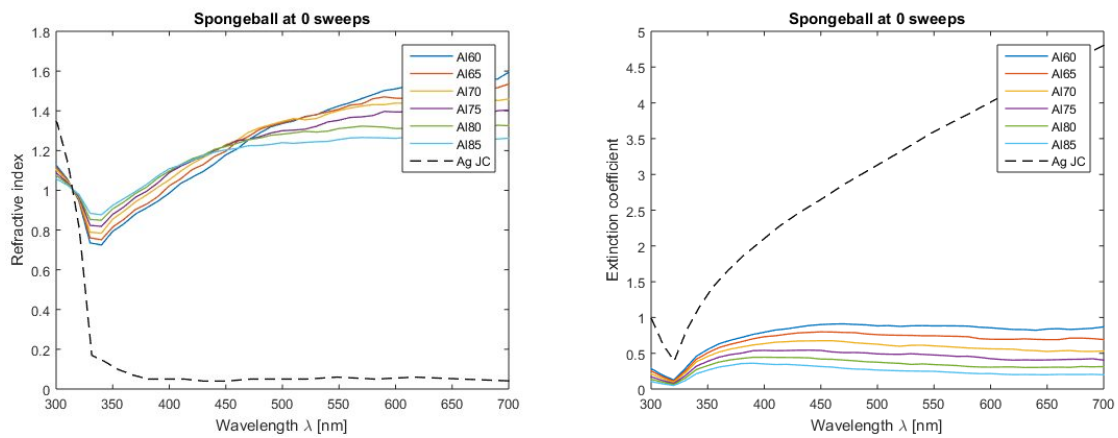

Figure 7. Comparison of the real part of the refractive index (left) and the extinction coefficient (k) (right) of the effective medium and the bulk silver (Johnson and Christy 1972).

\subsection{Morphology dependence}

We next examine whether the process of coarsening, as simulated by the annealing part of the sponge-making simulation, had an effect on the optical properties of the sponges. First, however, the possibility that the changes with annealing time might have been due to changes in the amount of Ag within each sponge sphere must be excluded. As explained previously, atomic movement takes place during the coarsening anneal, so changes in the optical properties could in principle have arisen from an increment of the silver concentration within the spherical volume. Therefore, to empirically prove the direct relation between sweeps and morphology, we calculated the Ag fill factors of every single sponge sphere. These are shown as a function of sweeps in Figure 8 and it is clear that there is negligible flux of Ag into or outside of the spherical simulation volume with time. Therefore, any variation of optical response from annealing must be due to change in the morphology.

The variation of optical extinction efficiency with time is shown in Figures 9 and 10 for the different type of sponges. In general, it can be seen that coarsening reduces the effective $Q_{e x t}$ of all of the sponges. As noted previously, the height of the extinction peak at about $400 \mathrm{~nm}$ is decreased as the void fraction increases. The data also suggest that there is a small red-shift in the peak position for the three high density sponges $(60,65$ and $70 \% \mathrm{Al}$ in the precursor) and a small shifted in the case of the low density sponges $(75,80$ and $85 \% \mathrm{Al}$ in the precursor). These changes are however at the limit of significance given the stochastic nature of the dealloying model. 


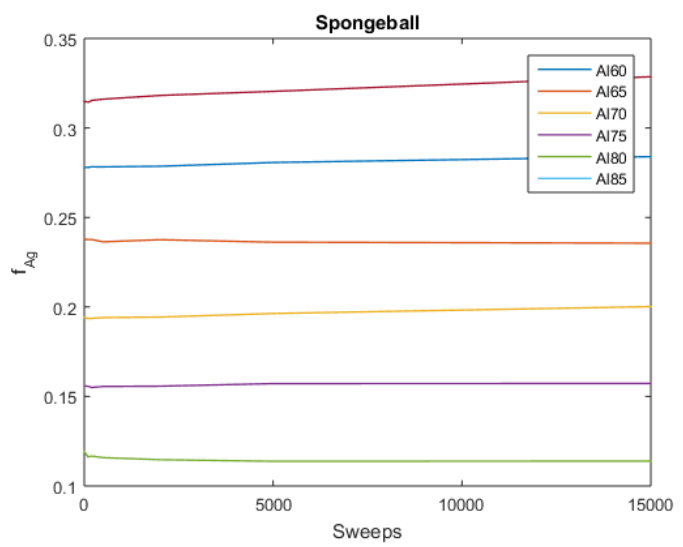

Figure 8. Representation of the small variation in the fill factor of silver due to the sweeps.

Finally, it is important to keep in mind that the annealing sweeps simulates the tendency of the sponge to attain a structural equilibrium at room temperature, i.e. more sweeps mean thicker and fewer ligaments. This change decreases the amount of light absorbed and hence decreases the extinction efficiency.
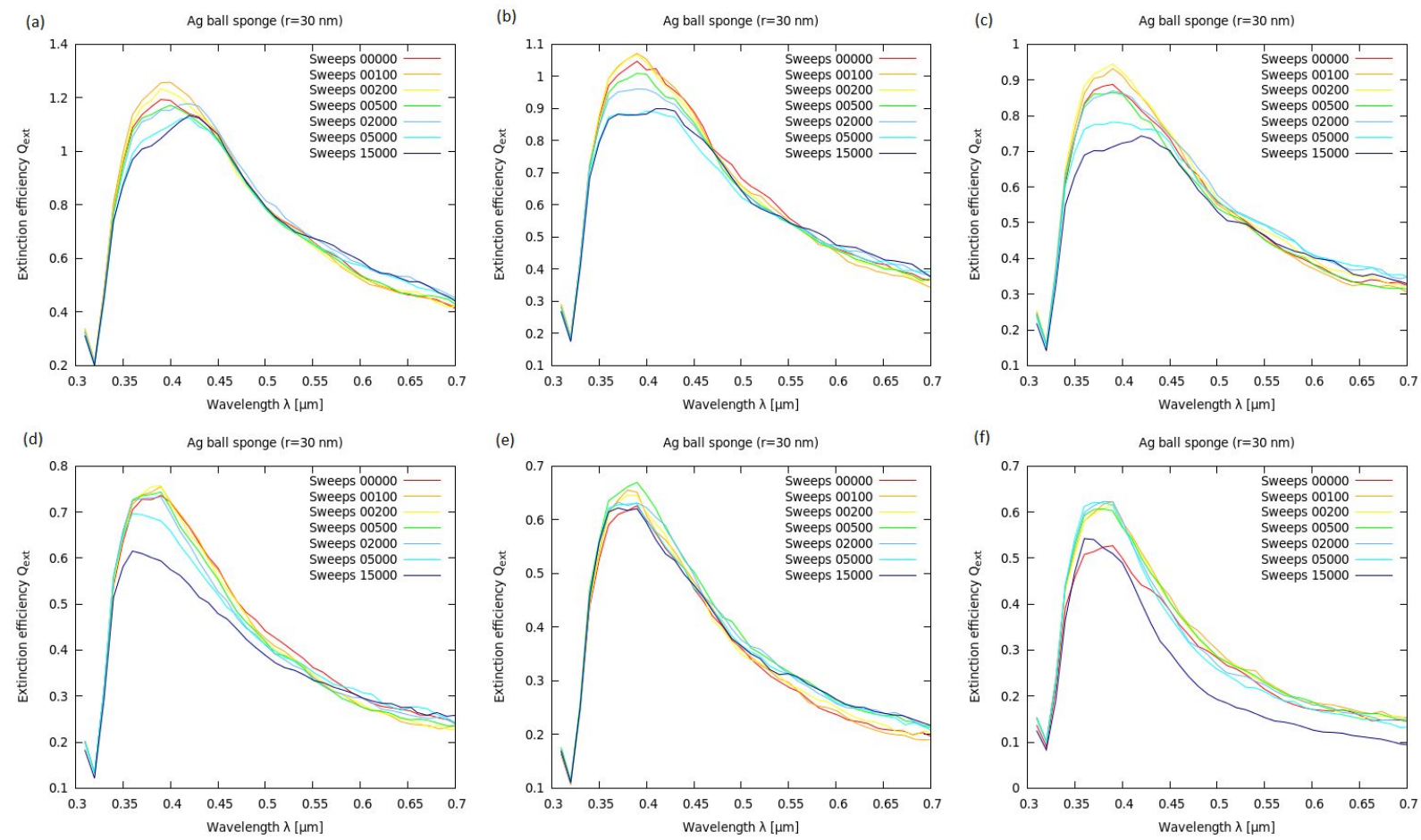

Figure 9. Change in the extinction efficiency at different sweeps of a sponge with an initial Al fill factor of (a) 60\%, (b) $65 \%$, (c) $70 \%$, (d) $75 \%$, (e) $80 \%$ and (f) $85 \%$.

The changes in the optical properties of the sponge spheres with elapsed annealing time cause associated changes in the effective medium model. An example is provided in Figure 11, which is for the sponge produced from the precursor with $60 \% \mathrm{Al}$. It is evident that the real part of the effective permittivity is always positive, but that it decreases as a result of the coarsening process, whereas the imaginary part increases slightly. These trends are most noticeable at the longer wavelengths. 


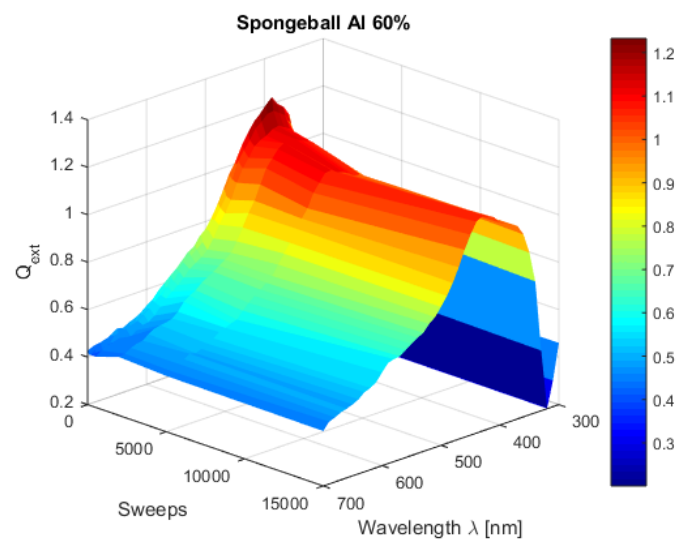

Figure 10. Study of the extinction efficiency of a spherical sponge in terms of the sweeps done.
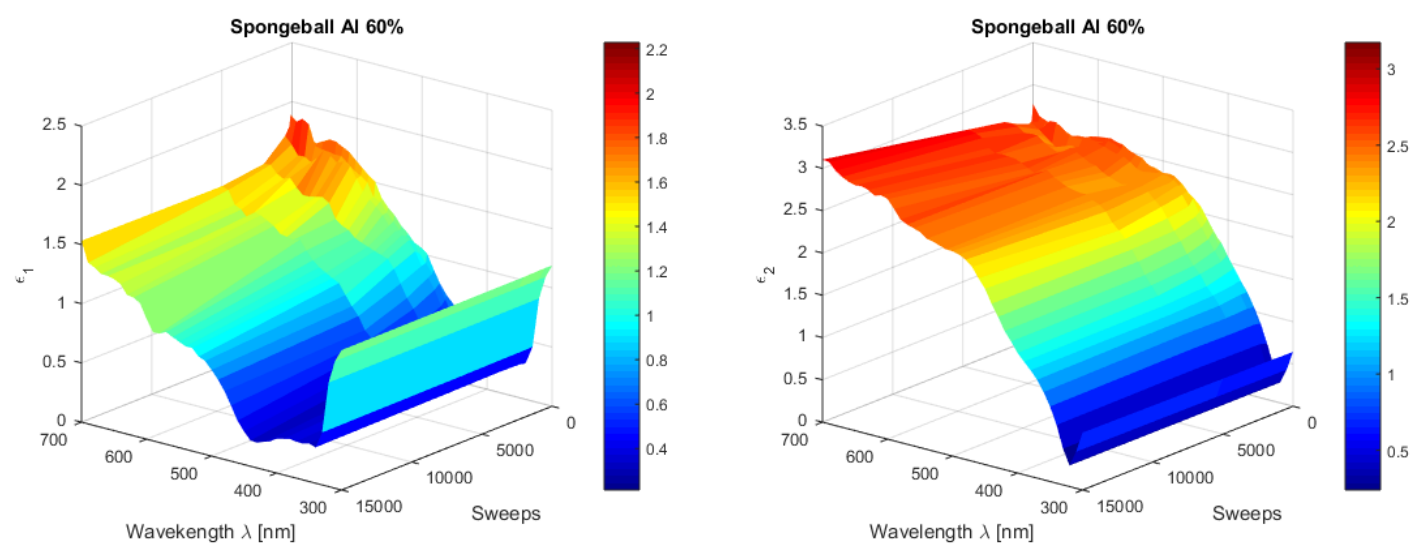

Figure 11. Real and imaginary parts of the permittivity of a sponge produced from a $60 \% \mathrm{Al}$ precursor.

\section{CONCLUSION}

In this project we used numerical methods to study the optical properties of model spherical targets made of nanoporous silver sponges. A range of simulated sponges of different densities and morphologies was investigated. A decrease in overall density of the silver reduced the absorption, but the spectral position of the absorption peaks was scarcely changed. The spherical shape of the targets was exploited to build an effective medium model for each type of sponge by solving the analytical Mie equations in reverse to extract an $\epsilon_{1}$ and $\epsilon_{2}$ for each wavelength. This model provided an effective refractive index of each sponge which incorporated the effects of void fraction and shape into an effective medium.

Analysis of the trends of the data indicates that, while fill fraction is the dominant factor controlling optical properties, the morphology of the sponge also has an important influence. As a result, we predict that it is possible to obtain wide range of effective media by fine tuning the morphology and density of silver sponges.

\section{REFERENCES}

[1] Smith, G. B., Maaroof, A. I., Allan, R. S., Schelm, S., Anstis, G. R., and Cortie, M. B., "Optical response of nanostructured metal/dielectric composites and multilayers," Proc. of SPIE 5508, 192-205 (2004).

[2] Galinski, H., Ryll, T., Schlagenhauf, L., Rechberger, F., Ying, S., Gauckler, L. J., Mornaghini, F. C. F., Ries, Y., Spolenak, R., and Döbeli, M., "Dealloying of platinum-aluminum thin Films: Dynamics of pattern formation," Physical Review Letters 107(22), 6-9 (2011). 
[3] Earp, A. A. and Smith, G. B., "Evolution of plasmonic response in growing silver thin films with prepercolation non-local conduction and emittance drop," Journal of Physics D: Applied Physics 44(25), 255102 (2011).

[4] Cortie, M. B., Maaroof, A. I., Stokes, N., and Mortari, A., "Mesoporous gold sponge," Australian Journal of Chemistry 60(7), 524-527 (2007).

[5] Garoli, D., Calandrini, E., Cattarin, S., Barison, S., Zilio, P., Bozzola, A., Toma, A., and De Angelis, F., "Engineered/tailored nanoporous gold structures for infrared plasmonics," Proc. of SPIE 9547, 95470K (2015).

[6] Smith, G. B., Maaroof, A., Dowd, A., Gentle, A., and Cortie, M., "Tuning plasma frequency for improved solar control glazing using mesoporous nanostructures," Proc. of SPIE 6197, 61970T (2006).

[7] Tai, M. C., Gentle, A., de Silva, K. S., Arnold, M. D., van der Lingen, E., and Cortie, M. B., "Thermal Stability of Nanoporous Raney Gold Catalyst," Metals 5(3), 1197-1211 (2015).

[8] Supansomboon, S., Porkovich, A., Dowd, A., Arnold, M. D., and Cortie, M. B., "Effect of precursor stoichiometry on the morphology of nanoporous platinum sponges," ACS Applied Materials and Interfaces 6(12), 9411-9417 (2014).

[9] Maaroof, A. I., Cortie, M. B., Gentle, A., and Smith, G. B., "Mesoporous gold sponge as a prototype 'metamaterial'," Physica B: Condensed Matter 394(2), 167-170 (2007).

[10] Jalas, D., Canchi, R., Petrov, A. Y., Lang, S., Shao, L., Weissmüller, J., and Eich, M., "Effective medium model for the spectral properties of nanoporous gold in the visible," Applied Physics Letters 105(24), 241906 (2014).

[11] Bergman, D. J., "Exactly solvable microscopic geometries and rigorous bounds for the complex dielectric constant of a two-component composite material," Physical Review Letters 44(19), 1285-1287 (1980).

[12] Draine, B. T., "The Discrete-Dipole Approximation and its Application to Interstellar Graphite Grains," The Astrophysical Journal 333, 848-872 (1988).

[13] Bohren, C. F. and Huffman, D. R., [Absorption and Scattering of Light by Small Particles] (2004).

[14] Maaroof, A. I., Gentle, A., Smith, G. B., and Cortie, M. B., "Bulk and surface plasmons in highly nanoporous gold films," Journal of Physics D: Applied Physics 40(18), 5675-5682 (2007).

[15] Lang, X., Qian, L., Guan, P., Zi, J., and Chen, M., "Localized surface plasmon resonance of nanoporous gold," Applied Physics Letters 98(9), 093701 (2011).

[16] Bosman, M., Anstis, G. R., Keast, V. J., Clarke, J. D., and Cortie, M. B., "Light splitting in nanoporous gold and silver," ACS Nano 6(1), 319-326 (2012).

[17] Burke, J. J., Stegeman, G. I., and Tamir, T., "Surface-polariton-like waves guided by thin, lossy metal films," Physical Review B 33(8), 5186-5201 (1986).

[18] Berini, P., "Plasmon-polariton waves guided by thin lossy metal films of finite width: Bound modes of symmetric structures," Physical Review B 61(15), 10484-10503 (2000).

[19] Arnold, M. D. and Blaber, M. G., "Optical performance and metallic absorption in nanoplasmonic systems," Optics express 17(5), 3835-3847 (2009). 